\title{
The Phenomenon of a Natural Thermonuclear Reactor
}

\author{
Andrzej Pawuła \\ Institute of Geology, Adam Mickiewicz University, Poznań, Poland \\ Email:pawula@amu.edu.pl
}

How to cite this paper: Pawuła, A. (2021). The Phenomenon of a Natural Thermonuclear Reactor. Journal of Geoscience and Environment Protection, 9, 92-109. https://doi.org/10.4236/gep.2021.92006

Received: December 31, 2020

Accepted: February 16, 2021

Published: February 19, 2021

Copyright (C) 2021 by author(s) and Scientific Research Publishing Inc. This work is licensed under the Creative Commons Attribution International License (CC BY 4.0).

http://creativecommons.org/licenses/by/4.0/

\begin{abstract}
The study contains the justification of J.M. Herndon's thesis about the existence of a thermonuclear reactor in the Earth's core. A scenario of the formation of a spontaneous nuclear reactor is presented, referring to the universal law of gravity, to the relationship between matter and energy, and to the properties of plasma. The theoretical attitudes of the discussed phenomena and the relations between them can be called the Theory of the Primordial Forces of Nature. It includes the interactions of the gravitational field, the forces of the electromagnetic field, which determine the properties of matter, and the strong interactions resulting from the release of nuclear energy. Arguments for the thesis about the commonness of the phenomenon of thermonuclear reactors in space are presented. The second part of the article presents examples of geological phenomena that confirm the activity of a thermonuclear reactor in the center of the Earth.
\end{abstract}

\section{Keywords}

Globe Evolution Cycle, Spontaneous Thermonuclear Reactor, Primary Forces of Nature Theory

\section{Helium Isotope ${ }^{3} \mathrm{He}$ as an Indicator of Thermonuclear Reactions}

The helium isotope ${ }^{3} \mathrm{He}$, formed in the proton-proton hydrogen cycle, is an indicator of the thermonuclear fusion reaction:

1) ${ }_{1}^{1} \mathrm{H}+{ }_{1}^{1} \mathrm{H} \rightarrow{ }_{1}^{2} \mathrm{H}+e^{+}+v_{e}$

2) ${ }_{1}^{2} \mathrm{H}+{ }_{1}^{1} \mathrm{H} \rightarrow{ }_{2}^{3} \mathrm{He}+\gamma$

3) ${ }_{2}^{3} \mathrm{He}+{ }_{2}^{3} \mathrm{He} \rightarrow{ }_{2}^{4} \mathrm{He}+{ }_{1}^{1} \mathrm{H}+{ }_{1}^{1} \mathrm{H}$

The detection of the ${ }^{3} \mathrm{He}$ helium isotope on the Sun was the basis for the conclusion that a nuclear reactor operates in it, and the source of solar radiation is ther- 
monuclear reactions (Dyson, Eddington, \& Davidson, 1920; Eddington, 1920, $1926,1928)$. The helium isotope ${ }^{3} \mathrm{He}$ has also been detected in ocean basalts in Hawaii and Iceland (Herndon, 1993, 2003, 2007) J. Marvin Herndon therefore concluded that this is evidence of a thermonuclear reactor in the Earth's core, where the basalt magma comes from. Even earlier, a team of oceanologists (Bearman et al., 1989) detected a zone of high concentration of helium ${ }^{3} \mathrm{He}$ isotope in ocean water, over submarine volcanoes. The presence of the helium ${ }^{3} \mathrm{He}$ isotope in volcanic gases and in the basalt magma from which volcanic gases are emitted is evidence of thermonuclear reactions, such as those in the Sun. The helium isotope ${ }^{3} \mathrm{He}$ is also formed in the Earth's atmosphere by the decay of tritium: but it is not related to the processes in the Earth's core. Hypothesis of the thermonuclear reactor with a uranium fuse was not convincing. In the opinion of physicists, the prevailing view was that in terrestrial conditions a thermonuclear reaction is impossible because the Earth's core is made up of elements that are too heavy, iron and nickel. It was also believed that the synthesis reactions took place only in a hydrogen gas medium and that under terrestrial conditions there was no adequately sealed reaction chamber.

It was believed that under the existing conditions, the Lawson criterion could not be met. The Lawson criterion is defined as the product of the density of matter $(\pi)$ and the necessary reaction time $(\tau)$, it is a variable quantity, depending on the individual properties of the reactants:

\begin{tabular}{lll}
\hline Lawson criterion & $\pi \cdot \tau \geq 10^{14} \mathrm{~s} \cdot \mathrm{cm}^{-3}$ & deuterium fusion-tritium \\
& $\pi \cdot \tau \geq 10^{16} \mathrm{~s} \cdot \mathrm{cm}^{-3}$ & deuterium-deuterium fusion \\
\hline
\end{tabular}

Herndon made the assumption that there is a uranium ball in the Earth's core and that the heat needed to trigger an avalanche thermonuclear reaction comes from uranium fission. Herndon used the analogy to the hydrogen bomb in which a uranium fuse was used to initiate an avalanche fusion reaction. The concept of a fusion georeactor with a uranium fuse has been rejected. In subsequent publications, Herndorn renewed his attempt to explain the phenomenon of Earth expansion and formulated a geological paradigm called "Whole Earth Decompression Dynamics (WEDD)". According to Herndon, the increase in the radius of the Earth is due to stored protoplanetary compression energy, magnified by uranium fission energy and radioactive decay energy. As the planetary volume increases, the surface area through rifting and the formation of global cracks increases. Basalt lava flows in the crevices, which, according to Herndon, moves by gravity along the surface. The hypothesis of the nuclear georeactor and the creation of basalt magma as the cause of the expansion of the Earth appeared olso in other publications (Pawuła, 1997a, 1997b, 2000a, 2000b).

\section{The Primordial Forces of Nature}

The role of cosmic rays as a factor responsible for the expansion of the Earth was 
discussed at the Institute of Nuclear Physics in Krakow and at the Laboratoire de Physique Subatomique et de Technologies Associèes SUBATECH in Nantes. The subject of matter creation and the existence of a georeactor was also presented at seminars at the Institute of Physics of the Warsaw University of Technology and the Polish Geological Society (Pawuła, 2000a). The prevailing view in the discussion was that cosmic rays are too weak a factor to justify the expansion of the Earth.

Only after twenty years, with sixty years of experience, the author of the article is convinced that he has found the key to solving the problem, i.e. the justification of the thesis about the thermonuclear reactor in the Earth's core. A thermonuclear reactor is the result of gravity, the physical properties of the plasma and the nuclides that make it up.

The primal phenomenon that connects matter with the energy hidden there is a gravity. Gravity is a physical phenomenon that we feel constantly. The importance of gravity in understanding the world in which we live is best reflected by the statement of an anonymous astrophysicist [quote from the website: http://www.fizykon.org/index.htm]: "The phenomenon of gravity is the binder of the Universe-it is the basic force operating between large bodies-celestial bodies in particular. It is a universal force, present in any corner of the cosmos. The discovery of gravity became an extremely important impulse for the intellectual development of mankind. The reason is that in the time before the discovery of this phenomenon, it was commonly believed that what is visible in the sky-stars, planets, the Sun, the Moon-are governed by completely different laws than objects from our immediate surroundings in science and philosophy-made it possible to look at the universe as a whole". A closer acquaintance with the phenomenon of gravity allows us to identify two basic effects of its operation: the gravitational increase in the mass of the globes and the formation of plasma. The increase in the mass of the globes is the result of the universal law of gravity and the mutual attraction of clusters of matter. The gravitational increase of the mass grows when the mass of the globs is growing (Koziar, 2018).

Plasma is ionized matter with special electromagnetic properties. There are basically two types of plasma, low temperature (cold) and high temperature (hot) plasma. Low-temperature plasma is formed at a lower ionization energy and is a magnetic trap that allows the formation of the walls of the reaction chamber that meets the Lawson criterion. Having a reaction chamber, tight and resistant to a nuclear explosion provided, a thermonuclear reactor spontaneously arises when the mass of the globe reaches a critical size (according to the author's assessment $10^{22} \mathrm{~kg}$ ). At a lower energy level, the ionization of matter consists in detaching electrons from the orbitals of atoms, at a higher energy level there is an interference inside the atomic nucleus. Low-temperature plasma begins to form at an ionization energy of $0.2 \mathrm{eV}$. The average kinetic energy of ionization of the lightest particles (electrons) is about $20 \mathrm{eV}$. The upper limit of the temperature of low-temperature plasma is several tens of thousands of degrees 
$\left.(20 \div 50) \cdot 10^{3} \mathrm{~K}\right)$. At very high temperatures $\left(>50 \times 10^{3} \mathrm{~K}\right)$, high-temperature plasma appears (Pluta, n.d.).

The origin of the elements is associated with thermonuclear reactions. If the existence of a georeactor and the formation of elements in the Earth's core are excluded, there remains an alternative view that the elements were formed in the reactions taking place during the Big Bang and in stars. The choice of the cosmological theory and the view on the origins of elements is important in the case of natural radioactive elements. One view is that global radioactivity is decreasing, but in fact the amount of radioactive elements is increasing. The discussion on the search for a real cosmological theory is therefore important in various fields. The Big Bang theory, assuming the creation of the universe, and therefore also the elements, in one explosion of a dimensionless "singularity" is rather a metaphysical speculation than a proof of the real world. Contrary to the promoted BB theory, which contradicts the laws of nature, the Theory of the Primordial Forces of Nature satisfies the logical requirements of describing physical phenomena. Despite the simplicity of the evolutionary diagram of the Earth and the solar system, detailed aspects of this process require clearing up apparent contradictions. An example of such a contradiction is the creation of matter and the expansion and defect of matter in thermonuclear reactions. A similar paradox concerns the burning out of stars and the increase in mass of all clusters of matter.

The answer to the remarks that deny the existence of the georeactor is the Theory of the Primary Forces of Nature (TPSN), which, using the natural laws of nature, explains the cause of geological phenomena, including the probable image of the Earth's core. The Theory of the Primordial Forces of Nature explains not only the creation of the Earth and the solar system, but also of the other globes of the cosmos. Taking into account the interaction of gravity, electromagnetic field forces and nuclear forces, the relationship of these forces with the mass of the globe, the density and properties of matter, and the energy hidden in matter was established. The action of the forces of nature explains the evolution of the Earth and the evolutionary cycle of the globe. Planets show varying degrees of heat, depending on their mass (Table 1). The fact is that there are hot planets and the thermal state of planets is related to their mass.

The dependence of the heat of the globes on their mass can be expressed by the thermal index WT, the percentage share of the hot core in the radius of the globe. For the Moon, this index is $9.5 \%$, for the Earth $44.5 \%$ and for Jupiter it is $100 \%$. The increase in temperature and pressure leads to the nuclide synthesis reaction. Theoretical calculations show that a spontaneous thermonuclear reaction takes place in a mass of $10^{30} \mathrm{~kg}$ of matter. When explaining the phenomenon of the Earth heating up, an important observation is the finding of the existence of cold and hot planets, and the relationship between the thermal state of the planets and their mass.

The logical conclusion is that the planets of the solar system can undergo a 
Table 1. Characteristics of selected planets of the solar system.

\begin{tabular}{|c|c|c|c|c|c|c|}
\hline & Mercury & Venus & Earth & Mars & Jupiter & Saturn \\
\hline diameter $[\mathrm{km}]$ & 4878 & 12,104 & 12,752 & 6788 & 142,800 & 120,660 \\
\hline $\begin{array}{l}\text { mass relative to } \\
\text { Earth mass }\end{array}$ & 0.05 & 0.815 & 1.0 & 0.107 & 317.9 & 95.1 \\
\hline density $\left[\mathrm{g} / \mathrm{cm}^{3}\right]$ & 5.43 & 5.24 & 5.52 & 3.04 & 1.32 & 0.7 \\
\hline atmosphere & lack & dense poisonous & $\begin{array}{c}\text { nitrogen } 79 \% \\
\text { oxygen } 21 \%\end{array}$ & $\mathrm{CO}_{2} 96 \%$ & $\begin{array}{l}\text { hydrogen and } \\
\text { helium }\end{array}$ & $\begin{array}{l}\text { hydrogen and } \\
\text { helium }\end{array}$ \\
\hline weak magnetic field & $\begin{array}{l}100 \text { times weaker } \\
\text { than Earth }\end{array}$ & weak & strong & fragmentary & Very strong & Very strong \\
\hline type of planet & rocky & rocky & rocky & rocky & $\begin{array}{c}\text { plasma } \\
\text { giant }\end{array}$ & $\begin{array}{l}\text { plasma } \\
\text { giant }\end{array}$ \\
\hline $\begin{array}{c}\text { thermal } \\
\text { classification }\end{array}$ & cold & cold & $\begin{array}{c}\text { cold } \\
\text { rift hot }\end{array}$ & cold & hot & hot \\
\hline
\end{tabular}

heating process analogous to that of the sun. On the Moon, with a radius of 1737 $\mathrm{km}$ and at a mass of $7.37 \times 10^{22} \mathrm{~kg}$, there is trace tectonic activity. Seismic surveys show the formation of a hot core with a radius of $165 \mathrm{~km}(9.5 \%$ of the radius of the globe). On the Moon, traces of volcanic eruptions in the form of basalt traps and periodic seismic tremors were observed, the hypocentres of which are located at a depth of $1000 \mathrm{~km}$, in the zone of the semi-liquid lower mantle.

Thermonuclear reactions appear in large clusters of matter, after exceeding the critical mass, when the force of gravity causes densification of matter and causes a corresponding increase in temperature. Due to the presence of a large number of ions of various charges, as well as free electrons, plasma strongly interacts with the electric and magnetic fields. For the same reasons, plasma is electrically conductive, and its resistance, unlike metals, decreases with increasing temperature. Plasma density increases because the occupied space has decreased after the removal of electrons from the orbits of atoms.

The factor facilitating the spontaneous formation of a thermonuclear reactor is the initial stage of low-temperature plasma production. It is only partially ionized plasma, but it has special electromagnetic properties. Eddy currents are induced in the plasma and then a magnetic field is created. The magnetized plasma layer forms the walls of the reaction chamber of the future reactor, ensuring the tightness condition required by the Lawson criteria. In the core of the Earth, the thickness of the magnetically stiffened sealing layer is $2000 \mathrm{~km}$.

In the process of the evolution of the globs, several stages can be distinguished, related to ionization and thermonuclear reactions. The boundary states of these stages are defined by the critical mass. The critical mass of $10^{22} \mathrm{~kg}$, corresponding to the mass of the Moon, concerns the initiation of thermonuclear reactions. The initial period between the initiation of thermonuclear reactions and the volcanic activity and the expansion of the globe is the period without plasma eruption. It is a long period of latent activity, without external forms of expansion of the globe. Only when the plasma pressure exceeds the gravitational 
pressure, the hot plasma is thrown outside the georeactor and transformed into basalt magma. The critical mass of $10^{30} \mathrm{~kg}$ corresponds to $1 / 2$ the mass of the Sun and is concerned with the transformation of a hot planet into a shining star. The critical mass of the globe of $10^{34} \mathrm{~kg}$ is associated with the collapse of the star and the closing of the globe's evolutionary cycle. The evolutionary sequence thus covers all globs, from as small and as cold as the Moon, to warm as Jupiter, to a hot star as the Sun and neutron stars.

The condition for the spontaneous formation of a thermonuclear reactor is the initial stage of ionization and the production of low-temperature plasma. Eddy currents appear in low-temperature plasma and generate a magnetic field. Low-temperature plasma, although only partially ionized, conducts electricity and is also subject to magnetization. These two properties make the layer of magnetically stiffened plasma create the right conditions for inducing eddy currents and a magnetic field. Due to these properties of matter, a thermonuclear reaction chamber is created spontaneously. Without taking this "detail" into account, the formation of thermonuclear reactors in space cannot be justified. Thus, thanks to the initial stage of low-temperature plasma production, the temperature in the center of the globe continues to rise and the heat energy is accumulated and not dissipated.

Ionization of matter is a phenomenon that leads to the formation of plasma, the nature of which depends on the energy level. Under low energy conditions, photoelectric effects appear, with higher ones-the Compton effect, scattering of gamma rays, and with even higher ones-the photon ceases to exist, interacting with matter and instead appears-a particle, an electron and its antiparticle: positron; the phenomenon of matter creation. Yarkovski (1888) already drew attention to the role of kinetic energy and gravity in the formation of chemical elements at the end of the $19^{\text {th }}$ century.

A characteristic feature of the cosmic matter, formed in the process of nucleosynthesis, i.e. hot plasma, is the presence of all nuclides from 1 to 94 and the abundance of light nuclides, hydrogen, helium, carbon, nitrogen and oxygen. The least amount is radioactive uranium and thorium, but their presence is stable. The domination, in hot plasma, of protons and helium nuclei results from the ease of participation of these nuclides in the synthesis reactions. Larger particles are also produced under stellar conditions. This is proven by experiments in heavy ion collision laboratories. Protons are also often a product of the collision of heavier nuclides, they are formed, for example, in the fusion of ${ }^{3} \mathrm{He}$ helium particles: ${ }_{2}^{3} \mathrm{He}+{ }_{2}^{3} \mathrm{He} \rightarrow{ }_{2}^{4} \mathrm{He}+{ }_{1}^{1} \mathrm{H}+{ }_{1}^{1} \mathrm{H}$.

The abundance of nuclides in the universe should not be interpreted as the composition of the nuclear fuel, but as the end product of the thermonuclear fusion process. By suggesting the dominance of light nuclides, especially hydrogen nuclei, it is assumed that the condition of a thermonuclear reaction should be a concentration of gaseous hydrogen. It is not hydrogen but the hydrogen nuclei that are simultaneously the fuel and the product of fusion reactions. Lawson's 
condition is met by every matter when the compressive force exceeds the resistance of the repulsive force and the tight housing of the reaction chamber is satisfied. Due to the action of gravity, electromagnetic and nuclear forces, there is an evolutionary sequence of globes, from planetesimals to rocky and hot planets to stars. The evolutionary sequence ends with a collapse of a massive star. High-temperature plasma is matter, $90 \%$ of which are protons.

The similar composition of the hot plasma of all stars, as well as of the Sun, proves that there are identical sources of plasma produced and the phenomenon of a thermonuclear reactor is common in the cosmos. Thermonuclear reactions appear in large clusters of matter, after exceeding the critical mass, when the force of gravity causes densification of matter and causes a corresponding increase in temperature. Theoretically, the automatic reaction of the synthesis of hydrogen atoms, under the influence of gravity, occurs with the mass of the hydrogen ball $10^{30} \mathrm{~kg}$ (1/2 the mass of the Sun). With a small mass of nuclear fuel, the nuclide synthesis reaction can take place in conditions of high temperature, of the order of millions of degrees, and a tight reaction chamber, ensuring that this temperature is maintained. The only material for building such a chamber is plasma, a mixture of ionized particles, negatively charged electrons and freely moving positive ions. Plasma is a good conductor of electric current, better than copper, and behaves like metal in a magnetic field. The reported critical mass of $10^{30} \mathrm{~kg}$ corresponds to the mass of young stars such as the sun. If we take into account the process of gravitational aggregation of the solar system globes from cold matter, we must add a preliminary stage, the Proto sun warming up, still as a rocky globe. The preliminary stage consists of the ionization of the matter and the production of low-temperature plasma, which will create tightness conditions for the reactor being formed. Increasing the gravitational pressure along with the increase in the mass of the globe causes a significant increase in temperature and the formation of successively-ions, atomic nuclei with a different number of nucleons, single-protons and neutrons, and finally quarks. The different ionization states of matter correspond to different phases of the plasma that differ in density. In the case of the plasma of the Sun's core, the plasma density is $1.6 \times 10^{2} \mathrm{~g} / \mathrm{cm}^{3}$, which is low compared to the density of the proton plasma of $10^{7} \mathrm{~g} / \mathrm{cm}^{3}$.

A magnetically stiffened insulating layer is created, a cover for the future reactor. At the same time, plasma, thanks to the tunneling effect, reduces the Coulomb forces between nuclides and facilitates their fusion. The fuel in thermonuclear reactions are particles with a different degree of ionization, from atoms with a changed configuration of electronic orbitals to proton and quark plasmas.

Taking the view that the solar system was formed from cold galactic matter, one should agree with the statement that the protosun was also a cold globe, like other globes. In the case of the Sun, due to its enormous mass, there was a rapid increase in temperature, the ionization of matter and the production of plasma, 
and finally thermonuclear reactions appeared. In the nuclide synthesis reaction, nuclear energy is released, corresponding to the difference between the masses of the reacting matter particles and the mass of the produced particles. This difference is called the mass defect. By analogy with the Sun, it can be concluded that a spontaneous thermonuclear reaction takes place in clusters of matter with a mass of $10^{30} \mathrm{~kg}$ (1/2 the mass of the Sun). The temperature must be very high, in the order of millions of degrees, and a sealed reaction chamber must be provided. A suitable material for the construction of such a chamber is plasma, a mixture of ionized particles, negatively charged electrons and freely moving positive ions. Plasma is a good conductor of electric current and behaves like metal in a magnetic field. Since the mass of the globe is constantly increasing due to gravity, the temperature of the globe is also increasing. The potential energy of gravitational pressure is transferred to particles of matter as kinetic energy (heat). In the synthesis reactions, part of the mass is converted into energy. The mass of the particle resulting from the synthesis is smaller than the sum of the mass of the reacting nuclides. The mass difference is radiated energy in the form of gamma electromagnetic waves.

As the mass of the globe and the force of gravity increase in the structure of the plasma, the share of light nuclides increases, with protons dominating. It is believed that with a matter density of $10^{7} \mathrm{~g} / \mathrm{cm}^{3}$, the plasma is homogeneous, proton. A further increase in gravitational pressure destroys the structure of the protons and forms a quark plasma with a density of $10^{14} \mathrm{~g} / \mathrm{cm}^{3}$. The extreme state of densification of matter ends with a collapse and a burst of gamma photons with enormous energy.

Ionization of matter begins with a kinetic energy of $0.2 \mathrm{eV}$. The electron removal process is complete when all orbital electrons are removed from the atoms. This is related to an ionization energy of about $2 \mathrm{MeV}$. The upper temperature limit of low-temperature plasma is tens of thousands of $\mathrm{K}$. After reaching the liquid state, low-temperature plasma acquires electromagnetic properties, conducts electricity and is magnetized. These two properties cause that the layer of magnetically stiffened plasma creates suitable conditions for the thermonuclear reaction chamber and also induces eddy currents generating the magnetosphere. Thus, due to the initial production stage of the shielding low temperature plasma, heat is accumulated. After reaching the critical mass of the globe, corresponding to the mass of the Moon, the reaction of the synthesis of ionized matter particles forming plasma is initiated.

Theory of the primary forces and the gravitational increase in mass applies to all matter clusters. In this evolution, the mass of the globe continues to increase, so gravitational pressure and temperature increase. Thermonuclear reactions take place in a highly concentrated medium of nucleons, sometimes called metallic hydrogen. The density of matter in the core of the Earth's reactor is estimated at $16 \mathrm{~g} / \mathrm{cm}^{3}$, in the solar reactor it is ten times higher and amounts to 160 $\mathrm{g} / \mathrm{cm}^{3}$. 
As the mass of the globe increases, the density and temperature in its center also increase. A symptom of the operation of a thermonuclear georeactor is the production of basalt magma and the expansion of the globe. Under the influence of the increasing plasma pressure, when the plasma pressure exceeds the gravitational pressure, the excess plasma is ejected to the zone D". The factor that strengthens the ionization of the Earth's matter is cosmic radiation.

It must be assumed that the thermonuclear reactions in the center of the Earth are analogous to those in the Sun. The induction of a thermonuclear reaction requires the production of high temperature, in the order of hundreds of millions of degrees, and a reaction chamber with sufficient strength to withstand very high pressure and extremely high temperature. Such conditions arise thanks to the magnetized protective layer.

\section{Gravity and the Evolutionary Cycle of Planets}

Gravity is a common phenomenon in Cosmos, and gravitational increase in mass affects all clusters of matter, from small planetesimals to giant stars. The increase in the mass of the globs, the ionization of matter and the increase in temperature, explain the transformation of cold globs into hot planets and stars. The above interpretation also explains the formation of double stars. Such a situation will arise in the solar system in a few million years, when the hot planet Jupiter will increase its mass and become a star

Spontaneous thermonuclear reactions occur in large clusters of matter, after exceeding the critical mass, when the force of gravity causes the matter to thicken and the temperature to rise accordingly. The force of gravity $F_{g}$ appears in the interaction of matter clusters and is expressed by the formula:

$$
F_{g}=G \frac{m_{1} \cdot m_{2}}{r^{2}}
$$

where: $G$ is the gravity constant $6.67 \times 10^{-11} N \mathrm{~m}^{2} / \mathrm{kg}^{2}, m$-mass of clusters of matter (globs), $r$-distance between the centers of gravity of the interacting globs.

High-temperature plasma is matter, the product of thermonuclear reactions, and constitutes $99 \%$ of the mass of the universe. A characteristic feature of the cosmic matter formed in the process of nucleosynthesis is the abundance of light nuclides, hydrogen, helium, carbon, nitrogen and oxygen. The least amount of radioactive material is uranium and thorium, but their presence does not change. The operation of the reactor is determined by the gravitational aggregation of space matter, ionization of concentrated matter and the production of plasma, which facilitates nuclide collisions through the tunneling phenomenon. From the beginning of the reactor's operation and the production of hot plasma, the latent activity period of the thermonuclear reactor begins. The plasma produced remains in the reactor, held back by a shielding layer of magnetically stiffened liquid plasma. Only when the plasma pressure exceeds the gravitational pressure does the phenomenon of plasma expansion and the expansion of the 
globe appear. In the process of the evolution of the globs, two sizes of the critical mass are distinguished. The first critical mass, of the order corresponding to the mass of the Moon, concerns the initial state of the hot core. Rift fissures and outflows of basalt magma appear, but there is no magnetic field yet. The second critical mass corresponding to the mass of the sun, concerns the transformation of the rocky globe into a star. A spontaneous thermonuclear reactor is created thanks to the production of an appropriate amount of plasma, even before the nuclide synthesis reaction occurs. The effect of thermonuclear reactions is nucleosynthesis. The most common elements include light elements with an atomic number below 10, i.e. mainly hydrogen, helium, carbon, nitrogen and oxygen. It should not be concluded from this that the fuel in thermonuclear reactors must be exclusively hydrogen nuclei. Heavier and larger particles in nuclear fuel require only more energy to overcome Coulomb resistances, they are less likely to participate in collisions and therefore there are fewer of them. The source of ionization and thermonuclear reactions energy, apart from gravitational pressure, is cosmic radiation and radiogenic heat.

In the center of the Sun, the plasma temperature is over 15 million degrees $\mathrm{K}$ and the density is $153 \mathrm{~g} / \mathrm{cm}^{3}$ [http://helio.astro.uni.wroc.pl/]. The solar thermonuclear reactor is in balance only because it does not burst under the pressure of the plasma because it is balanced by the force of the sun's gravity. Solar plasma, which has magnetic properties, is stopped by a magnetic field. If the plasma pressure is much higher than the gravitational pressure, the plasma is ejected from the globe in the form of prominence. The prominence consists of throwing plasma into outer space along with fragments of the frozen magnetic field, at a speed of up to $2000 \mathrm{~km} / \mathrm{s}$. Thermonuclear reactions take place only near the center of the sun, in an area extending to about 0.25 of the sun's radius from its center, i.e. within a radius of $174,065 \mathrm{~km}$. The sun is a typical star and an example of a spontaneous thermonuclear reactor formed in a cold rocky globe. The phenomenon of spontaneously forming thermonuclear reactors is common in the cosmos, so it also applies to planets and moons of the solar system.

The emergence of thermonuclear reactions on Earth can be associated with the Proterozoic era (3800 million years BP), when a magnetic field and volcanic activity appeared, an atmosphere composed of volcanic gases and the first primitive organisms appeared-proterio-bacteria. Initially, a low-temperature plasma was formed in the Earth's core. After the appearance of the magnetic field and telluric currents, the temperature of the Earth's core increased and nuclear reactions took place locally. Volcanic activity has significantly increased since the beginning of the Cambrian ( 560 million years BP) and this moment can be considered the formation of a global georeactor. An even stronger increase in geological activity, signaling the beginning of the Earth's expansion, occurred in Lower Perm, 280 million years BP. The production of basalt magma and its outflow through rift fissures increased. The traces of these eruptions are basalt covers "traps" in Perm deposits (250 - 280 million years BP). The oldest basalts that 
build ocean plates are dated at 180 million years. Hot plasma, ejected sporadically from the Earth's core, is the source material for basalt magma. Plasma discharges into zone $\mathrm{D}$ " occur when the plasma pressure exceeds the gravitational pressure. For comparison, in the center of the Earth, the gravitational pressure is $1.7 \times 10^{6} \mathrm{~atm}$., and the plasma pressure in the center of the Sun is $2.34 \times 10^{11}$ atm. After the plasma temperature in the $\mathrm{D}$ " layer is lowered, the process of completing the electron orbitals takes place.

High-temperature plasma is matter, the product of thermonuclear reactions, and constitutes $99 \%$ of the mass of the universe. A characteristic feature of the cosmic matter formed in the process of nucleosynthesis is the abundance of light nuclides, hydrogen, helium, carbon, nitrogen and oxygen. The least amount of radioactive material is uranium and thorium, but their presence does not change. The operation of the reactor is determined by the gravitational aggregation of space matter, ionization of concentrated matter and the production of plasma, which facilitates nuclide collisions through the tunneling phenomenon. From the beginning of the reactor's operation and the production of hot plasma, the latent activity period of the thermonuclear reactor begins. The plasma produced remains in the reactor, held back by a shielding layer of magnetically stiffened liquid plasma. Only when the plasma pressure exceeds the gravitational pressure does the phenomenon of plasma expansion and the expansion of the globe appear.

Gravity is a common phenomenon in Cosmos, and gravitational mass increase affects all clusters of matter, from small planetesimels to giant stars. Thus, the mass gain explains the existence of hot gas planets. In the process of creating matter, only the principle of conservation of energy and momentum must be met, but the principle of conservation of mass does not apply. In the process of the evolution of the globes, two sizes of the critical mass are distinguished. The first critical mass, of the order of $10^{22} \mathrm{~kg}$, corresponding to the mass of the Moon, concerns the initial state of the hot globe. Rift fissures and outflows of basalt magma appear, but there is no magnetic field yet. The second critical mass of $10^{30} \mathrm{~kg}$, corresponding to the mass of the sun, concerns the transformation of the rocky globe into a star. A spontaneous thermonuclear reactor is created thanks to the production of an appropriate amount of plasma, even before the nuclide synthesis reaction occurs. The effect of thermonuclear reactions is nucleosynthesis. The most common elements include light elements with an atomic number below 10, i.e. mainly hydrogen, helium, carbon, nitrogen and oxygen. It should not be concluded from this that the fuel in thermonuclear reactors must be exclusively hydrogen nuclei. Heavier and larger particles in nuclear fuel require only more energy to overcome Coulomb resistances, they are less likely to participate in collisions and therefore there are fewer of them. The source of ionization and thermonuclear reactions energy, apart from gravitational pressure, is cosmic radiation and radiogenic heat.

In the center of the Sun, the plasma temperature is over 15 million degrees $\mathrm{K}$ 
and the density is $153 \mathrm{~g} / \mathrm{cm}^{3}$ [http://helio.astro.uni.wroc.pl/]. The solar thermonuclear reactor is in balance only because it does not burst under the pressure of the plasma because it is balanced by the force of the sun's gravity. Solar plasma, which has magnetic properties, is stopped by a magnetic field. If the plasma pressure is much higher than the gravitational pressure, the plasma is ejected from the globe in the form of prominence. The sun is a typical star and an example of a spontaneous thermonuclear reactor formed in a cold rocky globe. The phenomenon of spontaneously forming thermonuclear reactors is common in the cosmos, so it also applies to planets and moons of the solar system.

The emergence of thermonuclear appeared on Earth can be associated with the Proterozoic era (3800 million years BP), when a magnetic field and volcanic activity appeared, an atmosphere composed of volcanic gases and the first primitive organisms appeared-proterio-bacteria. Initially, a low-temperature plasma was formed in the Earth's core. After the appearance of the magnetic field and telluric currents, the temperature of the Earth's core increased and nuclear reactions took place locally. The analysis of the course of seismic waves showed that the physical properties of the Earth's interior change at certain depths in a stepwise manner along the interface of phases with different velocity of propagation of elastic waves and different densities (Żelaźniewicz \& Grad, 2009). Volcanic activity has significantly increased since the beginning of the Cambrian (560 million years BP) and this moment can be considered the formation of a global georeactor. An even stronger increase in geological activity, signaling the beginning of the Earth's expansion, occurred in Lower Perm, 280 million years BP. The production of basalt magma and its outflow through rift fissures increased. The traces of these eruptions are basalt covers "traps" in Perm deposits (250 280 million years BP). The oldest basalts that build ocean plates are dated at 180 million years. Hot plasma, ejected sporadically from the Earth's core, is the source material for basalt magma. Plasma discharges into zone $\mathrm{D}$ " occur when the plasma pressure exceed.

\section{Examples of Thermonuclear Georeactor Operation}

\section{Example $1 .{ }^{3} \mathrm{He}$ helium isotope}

The presence of helium ${ }^{3} \mathrm{He}$ basalt and in ocean water over volcanoes, has been documented by J. M. Herndon and a team of oceanologists under the direction of G. Bearman. The ${ }^{3} \mathrm{He}$ helium isotope clearly indicates the operation of the georeactor in the center of the Earth.

Example 2. Basalt magma.

Basalt magma has special properties, is homogeneous, identical regardless of the place of outflow and has an elemental composition similar to solar plasma. Like the solar plasma, it contains a wide spectrum of rare elements and small but fixed amounts of uranium and thorium. An important criterion for determining the origin of basalts are radiometric studies of the radioactive series: ${ }^{238} \mathrm{U}$ uranium and ${ }^{232} \mathrm{Th}$ thorium. Radiogenic tests are a special type of birth record for 
the rocks examined. The age of basalts, forming ocean plates ranges from 0 to 180 million years B.P. This is unambiguous evidence of the contemporary formation of basalt magma. Basalt lava flows began in the period of Lower Perm (Autun), about 280,000 years by B.P. and are related to the phenomenon of Earth's expansion. The expansion process began with numerous cracks in the earth's crust and basalt magma outflows. Numerous basalt covers have been preserved on the continents, called "trap", including-Siberian traps and Central-Atlantic traps, as well as the European trap, in the NW part of Poland, at a depth of $3 \mathrm{~km}$.

The amount of basalt magma produced is an important proof of georeactor activity. Ocean plates, created by basalt magma, occupy over $70 \%$ of the globe. Geophysical surveys show not only the growth of oceanic plates in the surface zone but also the increase of the Earth's mantle, under the continental plates. The process of fusion reaction in the Earth's core is developmental, in the last period of 280 million years the radius of the Earth has increased, from 2800 to $6373 \mathrm{~km}$, and the radius of growth is an exponential function. Two hundred eighty million years ago, the volume of a small globe, with a radius of $2800 \mathrm{~km}$, was $9.2 \times 10^{10} \mathrm{~km}^{3}$, now with a radius of $6373 \mathrm{~km}$ it is $1.08 \times 10^{12} \mathrm{~km}^{3}$. Thus, the volume of the terrestrial globe increased by $9.88 \times 10^{11} \mathrm{~km}^{3}$. Over the last 280 million years, the volume of the earth has increased tenfold. Over the last 280 million years, the volume of the earth has increased tenfold. It can be concluded from this that during this period the georeactor produced plasma, from which $9.88 \times 10^{11} \mathrm{~km}^{3}$ of basalt magma was formed $\left(3.53 \times 10^{3} \mathrm{~km}^{3} /\right.$ year $)$. Part of this magma solidified on the surface of the terrain in the form of basalt rocks, and a much larger part crystallized in deep sea conditions, forming a series of magmatic rocks and emitting magmatic gases and helm solutions. The general conclusion is that magma rocks are less than 280 million years old and that the presented characteristics allow the association of basalt magma with hot plasma of a thermonuclear georeactor.

Example 3. Genesis of natural gas deposits.

In the Greater Poland Basin, natural gas deposits occur in Perm's deposits (Pawuła, 2017). At a depth of $3 \mathrm{~km}$, in the Autunu deposits basalt cover, a trace of Permian volcanism. According to stereotypical views, methane in natural gas is of organic origin. This is denied by the presence, in gas and in brine, of heavy metals and radioactive elements. In the study area, where the geothermal degree is $30 \mathrm{~m} / 1^{\circ} \mathrm{C}$, the helper zones and the corresponding depth zones are as follows (Table 2).

The magmatic solution is a dense condensate containing a whole range of elements from which elements selectively precipitate as salts or ores. The remaining molecules migrate further as brine components. The process of differentiation of magma and helper solutions causes an increase in the concentration of elements precipitating at low temperatures, mainly uranium and mercury. The concentrations of uranium are characteristic, low in meteorites and 
Table 2. Zones of igneous solutions.

\begin{tabular}{ccc}
\hline stage & temperature range & depth zone \\
\hline magma & $>1000^{\circ} \mathrm{C}$ & $>30 \mathrm{~km}$ \\
pegmatite & $600^{\circ} \mathrm{C}-1000^{\circ} \mathrm{C}$ & $18-30 \mathrm{~km}$ \\
pneumatolite & $400^{\circ} \mathrm{C}-600^{\circ} \mathrm{C}$ & $12-18 \mathrm{~km}$ \\
hydrothermal & $100^{\circ} \mathrm{C}-400^{\circ} \mathrm{C}$ & $3-12 \mathrm{~km}$ \\
\hline
\end{tabular}

basalts, very high in bituminous shale and phosphates:

- basalt rocks

$$
0.2 \div 0.8 \mathrm{ppm}
$$

- hard coal

$0.8 \div 1.5 \mathrm{ppm}$

- granite rocks

$2.8 \div 8 \mathrm{ppm}$

- bituminous shale

$9 \div 40 \mathrm{ppm}$

- phosphates

$25 \div 150$ ppm, incidentally 600 ppm.

At lower temperatures, magma gases and helper solutions $\left(\mathrm{CO}_{2}, \mathrm{H}_{2}, \mathrm{HCl}, \mathrm{H}_{2} \mathrm{~S}\right.$, $\mathrm{SO}_{2}, \mathrm{HF},\left((\mathrm{U}, \mathrm{Th}) \mathrm{O}_{2}\right)$. are released. In smaller quantities $\mathrm{CH}_{4}, \mathrm{NH}_{3}, \mathrm{P}_{4} \mathrm{O}_{10}$ are present. At low temperature, helper solution mercury compounds precipitate. Magmatic gases and thermal waters penetrate the surrounding rocks and form deposits of various elements. For example, crystallization of uranium oxides occurs only in the hydrothermal phase, below $400^{\circ} \mathrm{C}$. They release gases from cooling magma: hydrogen sulfide, carbon and sulfur oxides, nitrogen and hydrogen. Chemical reactions occur between these gases, especially the reactions of elemental carbon and carbon oxides with hydrogen, in which methane and water are formed:

$$
\begin{gathered}
\mathrm{C}+2 \mathrm{H}_{2} \rightarrow \mathrm{CH}_{4} \\
\mathrm{CO}+3 \mathrm{H}_{2} \stackrel{\mathrm{Ni}}{\longrightarrow} \mathrm{CH}_{4}+\mathrm{H}_{2} \mathrm{O}
\end{gathered}
$$

Inorganic methane can form deposits of gas enclosed in rock micropores due to the larger dimensions of the methane molecule than the reacting gas particles (pore trap). Juvenile water and migrating methane are components of brine. The natural gas deposit occurs in the Zechstein dolomite and Rotliegend sandstone, in an anti-claw trap, under layers of anhydrite and halite. The thickness of the salt series exceeds 1000 meters. Directly above the Rotliegend sandstones there is an ore layer, copper-bearing shale (Mansfeld level).

Hydrocarbons occur under a tight layer of salt, they do not have contact with the surface zone. High reservoir pressure excludes not only the possibility of moving deep into natural gas according to the subsidy scheme, but also excludes the process of leaching salt deposits by fresh water from the surface. The consequence of high reservoir pressure is the ascension of brine and bitumen traces at the surface of the site. Zechstein brine, at a depth of about 3 kilometers, contains, besides sodium chlorides, calcium and magnesium, gaseous nitrogen, carbon dioxide, hydrogen sulfide, hydrogen, helium and radon as well as heavy metals mercury and transuranides. Sodium chlorides as well as calcium and magnesium chlorides are obviously the dominant components in the deposit 
brine. A characteristic feature for Zechstein is the presence of hydrogen sulfide and anomalies of high reservoir pressures. The exceptionally high mineralization of brine and the presence of a specific element-bromine-is another proof of magmatic origin. The general physico-chemical formula of brine has no equivalent in atmospheric waters.

$$
\mathrm{Br}^{1,46-2,7} \mathrm{M}^{235-314} \frac{\mathrm{Cl}^{99}}{\mathrm{Na}^{51-66} \mathrm{Ca}^{28-46} \mathrm{Mg}^{0-6}} \mathrm{~T}^{101}
$$

where: $\mathrm{Br}$-bromine $\left(\mathrm{mg} \mathrm{Br} / \mathrm{dm}^{3}\right), \mathrm{M}$-mineralization $\left(\mathrm{g} / \mathrm{dm}^{3}\right)$, $\mathrm{T}$-temperature $\left({ }^{\circ} \mathrm{C}\right)$

At the level of the main dolomite (Zechstein, depth zone 3021 - $3051 \mathrm{~m}$ ) there is brine with extremely high mineralization, from 337 to $376 \mathrm{~g} / \mathrm{dm}^{3}$ ), with a high content of hydrogen sulfide. Nitrogenized gas is found (Table 3), it contains mercury $\left(0.045-0.230 \mathrm{mg} \mathrm{Hg} / \mathrm{dm}^{3}\right)$ and radon $\left(170 \mathrm{~Bq} \mathrm{Rn}-222 / \mathrm{m}^{3}\right)$ come from magma solutions.

\section{Analysis of radionuclides in brine from a natural gas deposit.}

Laboratory of Radiochemistry and Nuclear Metrology SMART, SUBATECH, Ecole des Mines de Nantes. Place of sampling: Piekary near Poznań, depth 2900 $\mathrm{m}$, Permian formations.

Radionuclides from the series were detected in the tested brine sample:

- uranium-radium: radium $\mathrm{Ra}-226$, lead $\mathrm{Pb}-214$, bismuth $\mathrm{Bi}-214$ and lead $\mathrm{Pb}-210$

- uranium-actinium: uranium U-235, radon Rn-219 and bismuth Bi-211.

- thorium: Ac-228 actinium, Ra-224 radium and $\mathrm{Pb}-212$ lead.

- separately occurring radioactive potassium K-40 isotope and mercury were detected.

However, no primary isotopes were detected in these series: uranium U-238 and Th-232 thorium.

Primary uranium and thorium radionuclides are absent in brine because they have precipitated at the optimum temperature for them at $100^{\circ} \mathrm{C}$, they are present in the sediment on the rocks. This confirms the thesis about the process of precipitation of metals and salts from brine. Uranium and mercury compounds persist in the brine, because the optimum temperature for uranium precipitation is about $100^{\circ} \mathrm{C}$, and for mercury $50^{\circ} \mathrm{C}$. The obvious source of brine are solutions and gases emitted from basalt magma, which is a product of a thermonuclear georeactor. At the bottom of the Zechstein deposits, in the layer of slate and

Table 3. Chemical composition of natural gas from the main dolomite (vol\%).

\begin{tabular}{cccccc}
\hline town & $\mathrm{CH}_{4}$ & $\mathrm{~N}_{2}$ & $\mathrm{H}_{2} \mathrm{~S}$ & $\mathrm{H}_{2}$ & $\mathrm{He}$ \\
\hline Zamysłowo & 12.88 & 85.51 & - & 1.597 & 0 \\
Będlewo & 82.81 & 13.78 & - & 3.399 & 0 \\
Swadzim & 88.13 & 9.85 & 1.4516 & 0.5882 & 0.0214 \\
Bolewice & 35.312 & 50.2077 & 4.013 & 0.0153 & 0.03 \\
\hline
\end{tabular}


Laboratory of Radiochemistry and Nuclear Metrology SMART, SUBATECH, Ecole des Mines de Nantes.

Place of sampling: Piekary near Poznań, depth 2900 m, Permian formations.

\begin{tabular}{|c|c|c|c|c|c|}
\hline \multicolumn{4}{|c|}{ 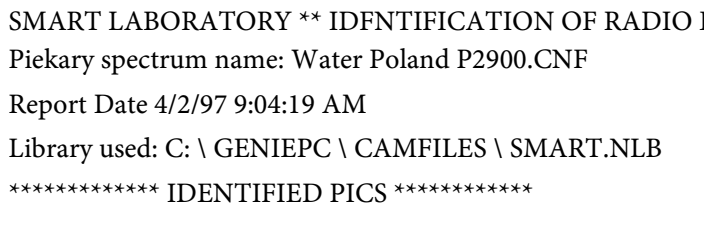 } & EMENTS ** SM & \\
\hline $\begin{array}{l}\text { Nuclide } \\
\text { Nom: }\end{array}$ & Id Confiance & Energie (keV) & $\begin{array}{c}\text { Emision } \\
(\%)\end{array}$ & Activité (Bq/1) & $\begin{array}{c}\text { Activité } \\
\text { Incertitude }\end{array}$ \\
\hline $\mathrm{K}-40$ & 0.971 & $1460.81^{\star}$ & 10.67 & $5.583 \mathrm{E} \div 01$ & $2.200 \mathrm{E}+00$ \\
\hline $\mathrm{Pb}-210$ & 0.977 & $46.54^{\star}$ & 4.10 & $3.190 \mathrm{E}+00$ & $1.469 \mathrm{E} \div 00$ \\
\hline BI-211 & 0.249 & $351.10^{*}$ & 12.20 & $6.397 \mathrm{E}+00$ & $7.042 \mathrm{E}-01$ \\
\hline PB-212 & 0.931 & $77.11^{\star}$ & 17.60 & $1.030 \mathrm{E}+00$ & $2.736 \mathrm{E}-01$ \\
\hline BI-214 & 0.680 & $964.08^{*}$ & 0.38 & $1.113 \mathrm{E}+01$ & $5.239 \mathrm{E}+00$ \\
\hline BI-214 & 0.680 & $1377.67^{*}$ & 3.92 & $3.023 \mathrm{E}+00$ & $3.514 \mathrm{E} \pm 00$ \\
\hline BI-214 & 0.680 & $1764.49^{*}$ & 15.36 & $3.024 \mathrm{E}+00$ & $1.104 \mathrm{E}+00$ \\
\hline PB-214 & 0.920 & $77.11^{\star}$ & 9.90 & $1.831 \mathrm{E} \div 00$ & $4.898 \mathrm{E}-01$ \\
\hline PB-214 & 0.920 & $295.21^{*}$ & 18.50 & $2.632 \mathrm{E} \div 00$ & $4.600 \mathrm{E}-01$ \\
\hline PB-214 & 0.920 & $351.92^{*}$ & 35.80 & $2.180 \mathrm{E}+00$ & $2.419 \mathrm{E}-01$ \\
\hline RA-224 & 0.922 & $240.99^{*}$ & 3.97 & $5.947 \mathrm{E}+00$ & $1.813 \mathrm{E}+00$ \\
\hline RA-226 & 0.989 & $186.21^{*}$ & 3.28 & $9.327 \mathrm{E}+00$ & $1.906 \mathrm{E}+00$ \\
\hline AC- 228 & 0.449 & $93.35^{*}$ & 1.12 & $5.760 \mathrm{E} \div 00$ & $3.121 \mathrm{E}+00$ \\
\hline AC-228 & 0.449 & $129.08^{*}$ & 2.93 & $5.446 \mathrm{E}-01$ & $1.684 \mathrm{E}+00$ \\
\hline AC- 228 & 0.449 & $209.26^{*}$ & 4.55 & $2.688 \mathrm{E} \div 00$ & $1.508 \mathrm{E}+00$ \\
\hline AC-228 & 0.449 & $270.27^{\star}$ & 3.77 & $1.751 \mathrm{E} \div 00$ & $1.659 \mathrm{E}+00$ \\
\hline AC- 228 & 0.449 & $338.37^{\star}$ & 12.00 & $1.851 \mathrm{E}+00$ & $6.062 \mathrm{E}-01$ \\
\hline AC-228 & 0.449 & $911.32^{\star}$ & 29.00 & $1.961 \mathrm{E} \div 00$ & $3.249 \mathrm{E}-01$ \\
\hline AC-228 & 0.449 & $969.16^{*}$ & 17.46 & $1.657 \mathrm{E}+00$ & $7.808 \mathrm{E}-01$ \\
\hline TH-234 & 0.260 & $92.80^{*}$ & 5.20 & $1.241 \mathrm{E}+00$ & $7.132 \mathrm{E}-01$ \\
\hline U-235 & 0.487 & $89.96^{*}$ & 1.50 & $-4.76 \mathrm{E}-01$ & $1.754 \mathrm{E}+00$ \\
\hline U-235 & 0.487 & $93.35^{*}$ & 2.50 & $2.580 \mathrm{E}+00$ & $1.398 \mathrm{E}+00$ \\
\hline U-235 & 0.487 & $185.71^{\star}$ & 54.00 & $5.666 \mathrm{E}-01$ & $1.161 \mathrm{E}-01$ \\
\hline
\end{tabular}

basic limestone, a few-meter-large mineralization zone was formed. In the mineralization zone: copper, zinc, lead, cobalt, molybdenum, nickel, gold, platinum and palladium. This indicates the penetration of roof rocks through the brine containing these metals, which is under high pressure. This proves the continuous activity of the process of precipitation of minerals from brine and the 
formation of a salt structure. It can be seen that the mineralization does not depend on the type of rock but is a subsequent process, depending on the penetration of the upwardly migrating brine. The salt floor layers sealing the Zechstein natural gas deposit are over a thousand meters thick. The copper mineralization zone concerns the copper shale layer and the bottom part of the basic limestone. They are not minerals formed from the evaporation of sea water, but compounds precipitating from the helper brine. The beginning of this process are thermonuclear reactions and the production of hot plasma, followed by the transformation of the plasma into basalt magma and the differentiation of magma and helper solutions.

Discussing the genesis of hydrocarbon deposits, adopting their organic or inorganic origin, is not just an academic dispute. The choice of the theory regarding the origin of methane in natural gas deposits has an impact on the efficiency of oil exploration. The effect of changing the view on the origin of methane can be seen in the success of US oil workers.

\section{Conclusion}

The sun is an example of a spontaneous thermonuclear reactor. The evolutionary cycle of the solar system began with the gravitational aggregation of cold galactic matter and its ionization. The effect of this process was the production of low-temperature plasma. After reaching critical mass, thermonuclear fusion reactions appeared, enabled by plasma electromagnetic and tunneling properties. Simultaneously with the creation of the georeactor, the production of hot plasma began. Under the influence of increasing plasma pressure, excess plasma is sent to the outer zone of the nucleus (layer D") and transformed there into basalt magma. The pressure of magma causes the expansion of the globe and the increase in mass of the Earth. Ultimately, the mass of hot plasma and basalt magma is created in the process of energy transformation, which we associate with the force of gravity!

\section{Conflicts of Interest}

The author declares no conflicts of interest regarding the publication of this paper.

\section{References}

Bearman, G., Brown, E., Coiling, A., Park, D., Phillips, J., Rothery, D., \& Wright, J. (1989). The Ocean Basins-Their Structure and Evolution. Milton Keynes: Walton Hall.

Dyson, F. W., Eddington, A. S., \& Davidson, C. (1920). A Determination of the Deflection of Light by the Sun's Gravitation Field, from Observations Made at the Total Eclipse of May 29, 1919. Philosophical Transaction of the Royal Society of London, 220, 291-333. https://doi.org/10.1098/rsta.1920.0009

Eddington, A. S. (1920). Report on the Relativity Theory of Gravitation. American Mathematical Monthly, 27, 129-130. https://doi.org/10.2307/2972740 
Eddington, A. S. (1926). The Internal Constitution of Stars. The Observatory, 43, 341-358.

Eddington, A. S. (1928). The Nature of the Physical World. New York, NY: The Macmillan Company; Cambridge: The University Press.

Herndon, J. M. (1993). Feasibility of a Nuclear Fission Reactor at the Center of the Earth as the Energy Source for the Geomagnetic Field. Journal of Geomagnetism and Geoelectricity, 45, 3423.

Herndon, J. M. (2003). Nuclear Georeactor Origin of Oceanic Basatl ${ }^{3} \mathrm{He} /{ }^{4} \mathrm{He}$, Evidence, a Implications. Proceedings of the National Academy of Sciences of the United States of America, 100, 3047-3050. https://doi.org/10.1073/pnas.0437778100

Herndon, J. M. (2007). Nuclear Georeactor Generation of Earth's Magnetic Field. Current Biography, 64, 45-49.

Koziar, J. (2018) 2018-Geological Proofs of Significant Expansion of the Earth. Wrocław: Association of Geologist Alumni of Wroclaw University.

Pawuła, A. (1997a). Are the Manifestations of Geological Activity a Consequence of Cosmic Rays? Paper at the Seminar of the Departments and Laboratory of High Energy Physics, Cracow: H. Niewodniczański Institute of Nuclear Physics.

Pawuła, A. (1997b). Contribution à l'explication des anomalies $d u$ radon $R n-222$ dans le milieu naturel. Nantes: Seminaire SUBATECH, Laboratoire de Physique Subatomique et de Technologies associèes.

Pawuła, A. (2000a). Evolution of the Earth in the light of GPS Measurements (pp. 25-38). Papers, T.IX, Poznań: Polish Geological Society-Institute of Geology, Adam Mickiewicz University.

Pawuła, A. (2000b). Geological Conditions of Radon Anomalies. "Radon in the Environment” Scientific Session. Krakow: Institute of Nuclear Physics.

Pawuła, A. (2017). Hydrogeology of Central Wielkopolska. Poznań: Bogucki Scientific Publishers.

Pluta, J. (n.d.) Website of the Institute of Physics, Warsaw University of Technology. http://www.fizyka.pw.edu.pl/index.php/pl/

Yarkovski, J. (1888). Hypothese cinetique de la gravitation universelle en connexion avec la formation des elements chimiques. Moscow.

Żelaźniewicz, A., \& Grad, M. (2009). What Is Happening inside the Earth? Warsaw: Planet Earth Committee of the Polish Academy of Sciences, 7th Department of the Polish Academy of Sciences. 